\title{
RGB color coded images in scanning electron microscopy of biological surfaces
}

\author{
O. KOFROŇOVÁ, O. BENADA*
}

Institute of Microbiology of the CAS, v.v.i., Vídeňská 1083, 14220 Prague 4, Czech Republic

\begin{abstract}
Summary. - We present here a methodological approach for the creation of color images in scanning electron microscopy by processing grayscale images taken simultaneously from at least three different detectors in a scanning electron microscope. The final color images are then produced by merging together those grayscale images in RGB color space. We show the images from non-conductive standard sample together with those obtained from real microbiological samples. The first one represents a microbial biofilm naturally grown on fiber glass filter. The other shows individual Bacillus subtilis cells from batch culture. All the image handling was done in open source image processing software ImageJ or GNU Image Manipulation Program (Gimp) or, alternatively, in proprietary AnalySis 3.2 Pro software processing suite.
\end{abstract}

Keywords: biological surfaces; color images; high resolution; scanning electron microscopy

\section{Introduction}

High resolution surface investigation is necessary for microbiology when bacterial cell surface details have to be studied. Bacterial flagella or pili are of the size that conventional scanning electron microscope is hardly, if ever, able to show. The resolution achieved in field emission gun scanning electron microscopes (FEG-SEM) is generally of the order of nanometers and can reach subnanometer order in special devices (XHR-SEM, Roussel et al., 2009). The field emission gun electron sources and newly developed detectors used in the modern scanning electron microscopes thus provide image resolution that is interesting for microbiologists. The FEG-SEM in combination with backscattered electron detectors offers the opportunity to show these details on uncoated or slightly coated samples with $3 \mathrm{~nm}$ of platinum. The low-voltage mode of FEG-SEM in combination with beam deceleration mode gives sufficient contrast (Phifer et al., 2009) with an advantage of an angle selective detection of backscattered electrons using a concentric backscattered detector (CBS, Wan et al. 2016), even on uncoated biological samples. However, the biological sample resolution essen-

*Corresponding author. E-mail: benada@biomed.cas.cz; phone: +420-2-41062399.

Abbreviations: $\mathrm{CBS}=$ concentric backscattered detector or circular back scattered detector; FEG-SEM = field emission gun scanning electron microscopy; TLD = through the lens detector, TLD$\mathrm{CN}=$ charge neutralization mode tially depends on sample preparation procedure (Fischer et al., 2012). Fine details on bacterial cell surface can be detected and described on properly prepared samples, e.g. the positions of z-ring on Streptococcus pneumoniae mutants and wild type (Ulrych et al., 2016).

The images from scanning electron microscopes are usually in grayscale. The first attempts to get colored scanning electron micrographs can be dated to the beginning of the seventies (Swift and Brown, 1975) to the "analog era" in SEM imaging. Then there were many attempts to develop methods for proper coloring of grayscale SEM images see e.g. Oho and Watanabe (2001), for review see Sim et al. (2008).

Nowadays, the different high resolution detectors that are available for SEM offer an opportunity to encode their signals in a red, green and blue channel and produce 24-bit color images (Diller, 2010; Kinsmann, 2014). Here we show some applications of RGB color coding of grayscale images recorded simultaneously using secondary (through the lens detector; TLD) and backscattered electron (CBS) detectors in the investigation of biological samples mainly of microbiological origin.

\section{Material and Methods}

Sample preparation. The meadow brown (Maniola jurtina; Linnaeus, 1758) wing scales were used as a standard sample. A part of the air dried butterfly wing was glued onto aluminum stub 
using silver conductive paint and used without any coating. Bacillus subtilis strain and fiber glass filter with natural biofilm were obtained from the Institute of Microbiology CAS v.v.i. research groups. The bacteria cultures were fixed with $3 \%$ glutaraldehyde in cacodylate buffer overnight at $4^{\circ} \mathrm{C}$ and extensively washed with cacodylate buffer. The washed cells were then allowed to sediment onto $12 \mathrm{~mm}$ round glass coverslips treated with poly-L-lysine at $4^{\circ} \mathrm{C}$ overnight. Next day the coverslips with attached bacteria were dehydrated through alcohol series and critical-point dried from liquid $\mathrm{CO}_{2}$ in a K850 Critical Point Dryer (Quorum Technologies Ltd, Ringmer, UK). Finally, the dried coverslips were sputter-coated with $3 \mathrm{~nm}$ of platinum in a Q150T ES sputter coater (Quorum Technologies Ltd.). The fiber glass filter with natural biofilm was fixed also with $3 \%$ glutaraldehyde in cacodylate buffer overnight at $4^{\circ} \mathrm{C}$, extensively washed with cacodylate buffer and dehydrated as described above. A part of the dried glass filter approximately $10 \times 10 \mathrm{~mm}$ was glued onto standard aluminum stubs with colloidal silver paint (leitsilber) and sputter-coated with $3 \mathrm{~nm}$ of platinum.

Scanning electron microscopy. The final samples were examined in the FEI Nova NanoSEM 450 (FEI, Brno, Czech Republic) scanning electron microscope using SE, TLD and CBS detectors at accelerating voltage ranging from 1 to $5 \mathrm{kV}$ and beam deceleration mode $(0.2$ to $2 \mathrm{kV})$. The specific details of image recording conditions are given in the corresponding figure legends.

Software. Free open source software ImageJ (Schneider et al., 2012) or its distribution Fiji (Schindelin et al. 2012), open source Gimp (https://www.gimp.org) and a proprietary software AnalySis3.2 Pro (EMSIS GmbH, Germany) were used in the study.

\section{Results and Discussion}

The CBS backscattered electrons detector of Nova NanoSEM scanning electron microscope is useful for problematic samples when charging and edge effects play an important role in image deterioration. With the aid of the beam deceleration mode (Phifer et al., 2009) it may be possible to find out specific imaging conditions, when TLD secondary electron detector can be used for image recording without affecting the recorded image by sample charging artifacts. Therefore, we used the possibility to simultaneously record the signal from A, B and C segments of the CBS detector together with the secondary electrons signal from the TLD detector. Alternatively, when charging was a problem, a signal from $\mathrm{D}$ segment of the CBS detector was recorded instead of the signal from the TLD detector. Thus, we obtained a set of four grayscale images for each sample. This set of images gives the possibility to use several triplet combinations for creating final RGB color image. The main criterion for three images subset selection was the proper enhancement of specific surface detail(s) in the final 24-bit color image.

Butterfly wing scales are made up of a chitin, an organic compound consisting of long polymeric chains of $\mathrm{N}$-acetylglucosamine with characteristic submicrometer texture. Uncoated samples of butterfly wing scales serve us as a standard for setting up the conditions for uncoated non-conductive surface imaging using the TLD and the CBS detectors. The typical images taken with different segments

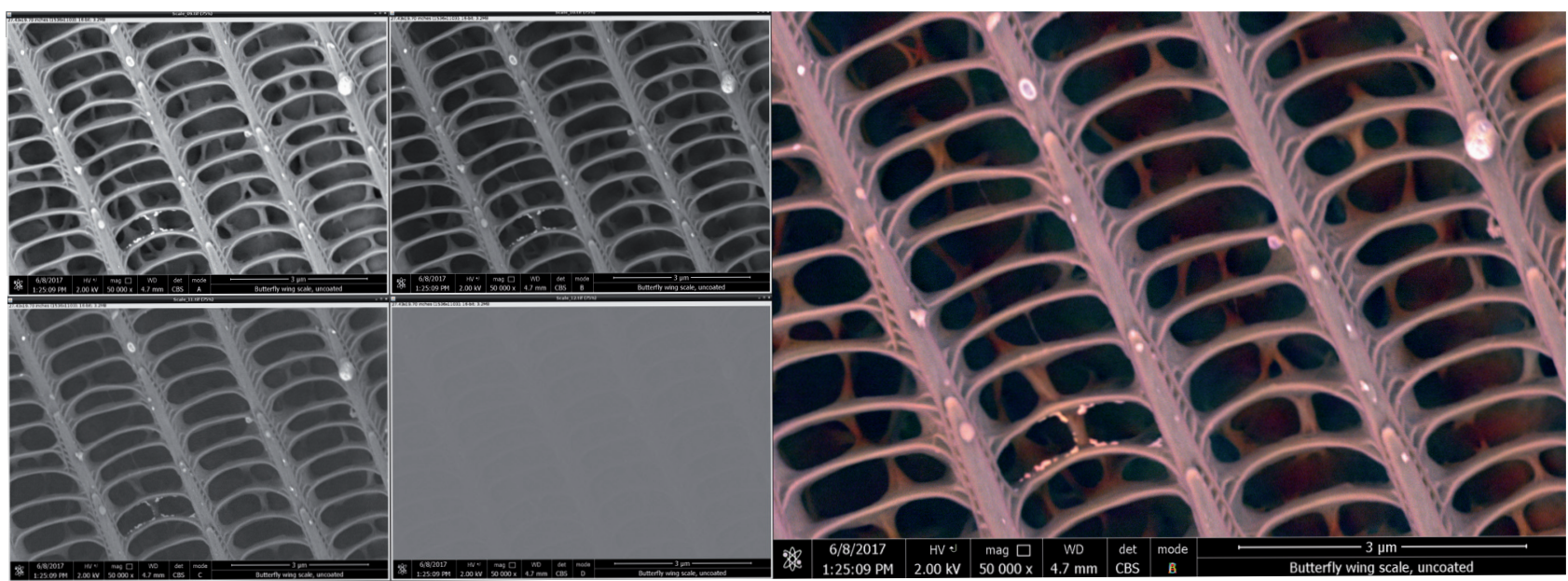

Fig. 1

Standard sample of Maniola jurtina wing scale imaged using CBS detector at $2 \mathrm{kV}$ (electron beam landing energy) and beam deceleration of $1037.5 \mathrm{~V}$ (stage bias)

The images from individual segments are shown in left part of the image: quadrant $1=\mathrm{CBS}-\mathrm{A}$ segment, quadrant $2=\mathrm{CBS}-\mathrm{B}$ segment, quadrant $3=\mathrm{CBS}-\mathrm{C}$ segment and quadrant 4 =CBS-D segment. The signal from D segment of the CBS detector was low with a lot of noise, therefore it was not chosen for final color image processing. In the right part of the image, there is a final color image created by RGB coding of grayscale images recorded from the CBS detector. RGB coding scheme: $\mathrm{R}=\mathrm{CBS}-\mathrm{A}$ segment, $\mathrm{G}=\mathrm{CBS}-\mathrm{B}$ segment and B = CBS-C segment. An ImageJ processing. 


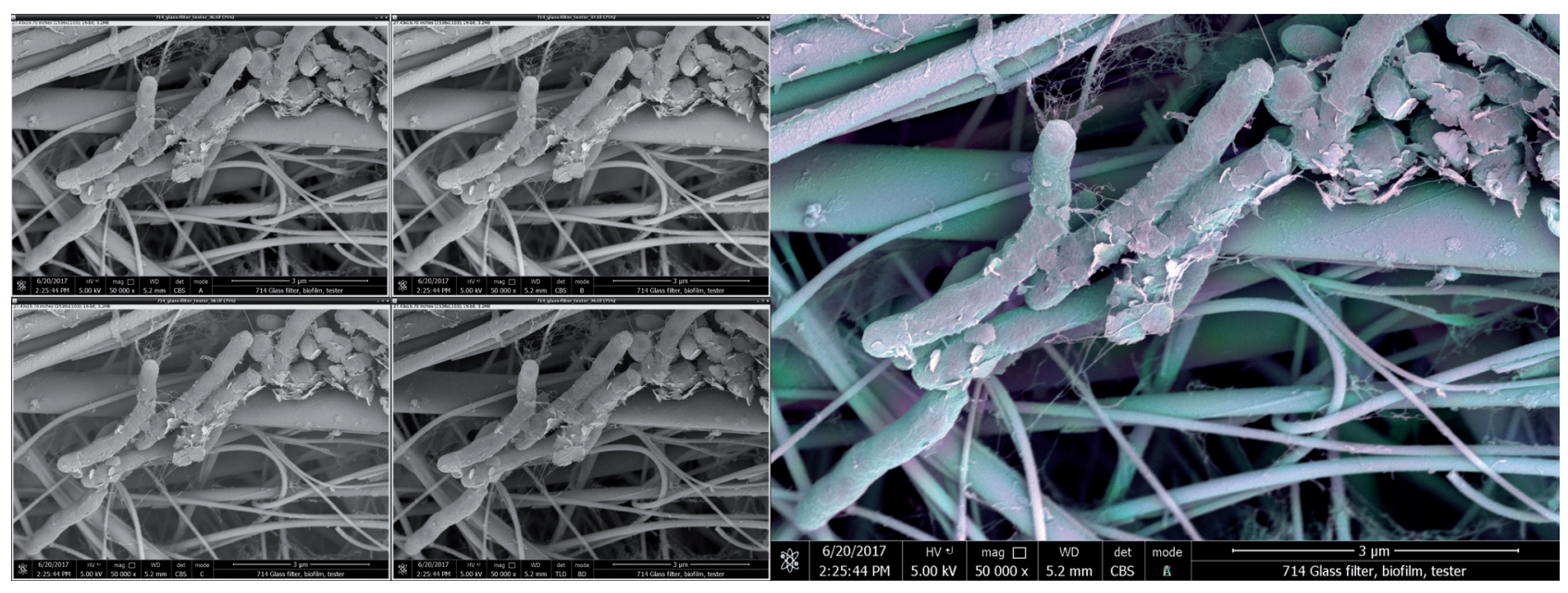

Fig. 2

Bacterial biofilm on the fiber glass filter imaged with the CBS and the TLD detectors at $5 \mathrm{kV}$ (electron beam landing energy) and beam deceleration of $1037.5 \mathrm{~V}$ (stage bias)

The images from the CBS and the TLD detectors are in the left part of the image: quadrant $1=$ CBS-A segment, quadrant $2=$ CBS-B segment, quadrant $3=\mathrm{CBS}-\mathrm{C}$ segment and quadrant $4=$ TLD-CN. The final color image is on the right. The RGB coding scheme: $\mathrm{R}=\mathrm{CBS}-\mathrm{C}$ segment, $\mathrm{G}=\mathrm{CBS}-\mathrm{A}$ segment and $\mathrm{B}=\mathrm{CBS}-\mathrm{B}$ segment. Undetermined bacteria were detected on glass fibers of diverse thickness. The collapsed extracellular polymeric substance and bacterial pili-like structures are nicely enhanced in the color image. An ImageJ processing.
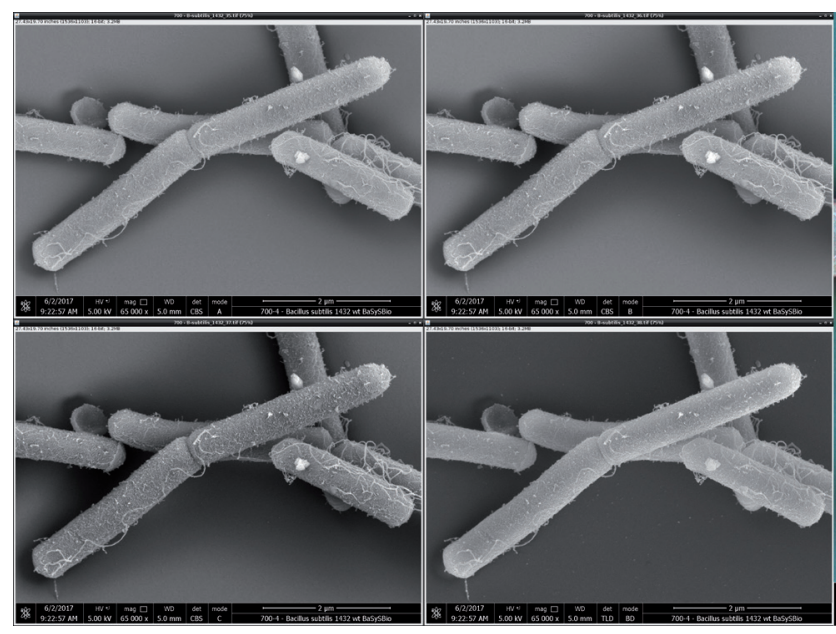

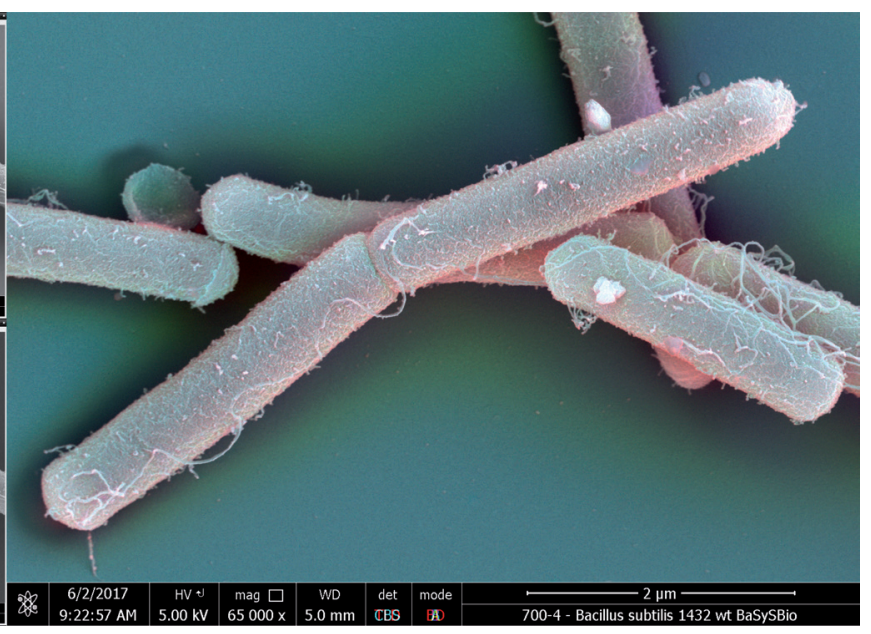

Fig. 3

Bacterial cells of Bacillus subtilis from exponential growth phase imaged with the CBS and the TLD detectors at $5 \mathrm{kV}$ (electron beam landing energy) and beam deceleration of $1432.5 \mathrm{~V}$ (stage bias)

The images from the CBS and the TLD detectors are in the left part of the image: quadrant $1=$ CBS-A segment, quadrant $2=$ CBS-B segment, quadrant $3=$ CBS-C segment and quadrant $4=$ TLD-CN. The final color image is on the right. The RGB coding scheme: $\mathrm{R}=\mathrm{TLD}-\mathrm{CN}, \mathrm{G}=\mathrm{CBS}-\mathrm{A}$ segment and $\mathrm{B}=$ CBS-B segment. Fine flagella-like or pili-like structures enhanced in light color are shown of the bacterial surfaces. An ImageJ processing.

of the CBS detector are shown in Fig 1. Unfortunately, we were not able to determine beam deceleration settings for simultaneous recording of backscattered and secondary electrons in the CBS and the TLD detectors. In this case only the CBS detector imaging was used. For RGB color coding we select the signal from $\mathrm{A}, \mathrm{B}$ and $\mathrm{C}$ segments of the CBS detector, because the signal from the CBS-D segment was weak and noisy (Fig. 1, quadrant 4).

A slight metal coating with $3 \mathrm{~nm}$ of platinum was used for real microbiological samples. We selected a fiber glass filters 
with naturally developed microbial biofilms and batch cultured cells of Bacillus subtilis. Fig. 2 gives an example of the following RGB coded images: $R=$ CBS- $C$ segment, $G=C B S-A$ segment and $\mathrm{B}=\mathrm{CBS}-\mathrm{B}$ segment. Undetermined bacteria forming microbial biofilm on glass fibers of diverse thickness are shown. The final RGB image clearly shows the collapsed extracellular matter and bacterial pili-like structures.

Fig. 3 is an example of $B$. subtilis cells from exponential growth phase. RGB coding of the final image is the following: $\mathrm{R}=\mathrm{TLD}-\mathrm{CN}, \mathrm{G}=\mathrm{CBS}-\mathrm{A}$ segment and $\mathrm{B}=\mathrm{CBS}-\mathrm{B}$ segment . Fine flagella-like or pili-like (fimbriae) structures on bacteria surface are nicely enhanced in the color image.

All the image processing of presented images was done in ImageJ (version 1.51o). Original grayscale SEM images were recorded at 16-bit grayscale. Using of ImageJ was therefore advantageous as it can process 16-bit grayscale images directly without converting them to the 8-bit grayscale. However, 16-bit grayscale images had to be converted to the 8-bit grayscale when they were processed in the Gimp (version 2.8.14), because the Gimp 2.8 still does not handle 16-bit grayscale images properly. The same processing can be done in the AnalySis3.2 image processing suite with the advantage of powerful macro image processing instructions. However, AnalySis3.2 is a non-free software.

\section{Conclusion}

We have shown here an easy procedure how to enhance surface details in SEM images in colors. For high resolution work, it is necessary to record images from different detectors at once in order to minimize the effect of image drift. This can easily occur when non-conductive or slightly conductive samples, the most of biological ones, have to be imaged. We showed that with RGB color coding of grayscale image sets it is possible to create color images with enhanced details, because they combine an information obtained from three different detectors.

Acknowledgements. We would like to thank M. Grydler for supporting us with bacterial biofilms, J. Pospíšil for Bacillus subtilis culturing and Z. Žižka for butterfly scales. All collaborators are from the Institute of Microbiology of the CAS, v.v.i. The support from Ministry of Education, Youth and Sports of the Czech Republic (LO1509) and Czech Science Foundation (16-20229S) is greatly acknowledged.

\section{References}

Diller S (2010): Wie kommt die Farbe in das rasterelektronenmikroskopische Bild? Mikrokosmos 99, 367-371.

Fischer ER, Hansen BT, Nair V, Hoyt FH, Dorward DW (2012): Curr. Protoc. Microbiol. 25, B:2B.2:2B.2.1-2B.2.47, Scanning Electron Microscopy, John Wiley \& Sons, Inc. https://doi.org/10.1002/9780471729259.mc02b02s25

Kinsmann T (2014): A Technique for Generating Colour Scanning Electron Microscope Images. InFocus 34, 24-43.

Oho E, Watanabe M (2001): Natural color scanning electron microscopy based on the frequency characteristics of the human visual system. Scanning 23, 24-31. https://doi. org/10.1002/sca.4950230104

Phifer D, Tuma L, Vystavel T, Wandrol P, Young R (2009): Improving SEM imaging performance using beam deceleration. Microsc. Today 17, 40-49. https://doi.org/10.1017/ $\underline{\text { S1551929509000170 }}$

Roussel LY, Stokes DJ, Gestmann I, Darus M, Young RJ (2009): Extreme high resolution scanning electron microscopy (XHR SEM) and beyond. Proc. SPIE 7378, Scanning Microsc. 7378W. https://doi.org/10.1117/12.821826

Schindelin J, Arganda-Carreras I, Frise E, Kaynig V, Longair M, Pietzsch T, Preibisch S, Rueden C, Saalfeld S, Schmid B, Tinevez J-Y, White DJ, Hartenstein V, Eliceiri K, Tomancak P, Cardona A (2012): Fiji: an open-source platform for biological-image analysis. Nat. Methods 9, 676-682. https://doi.org/10.1038/nmeth.2019

Schneider CA, Rasband WS, Eliceiri KW (2012): NIH Image to Image): 25 years of image analysis. Nat. Methods 9 , 671-675. https://doi.org/10.1038/nmeth.2089

Sim KS, Tso CP, Ting HY (2008): Canny optimization technique for electron microscope image colourization. J. Microsc. 232, 313-334. https://doi.org/10.1111/j.1365-2818 $.2008 .02103 . \mathrm{x}$

Swift JA, Brown AC (1975): A technique for obtaining scanning electron micrographs in colour. J. Microsc. 105, 1-14.

Ulrych A, Holečková N, Goldová J, Doubravová L, Benada O, Kofroňová O, Halada P, Branny P (2016): Characterization of pneumococcal Ser/Thr protein phosphatase phpP mutant and identification of a novel PhpP substrate, putative RNA binding protein Jag. BMC Microbiology 16, 247. https://doi.org/10.1186/s12866-016-0865-6

Wan Q, Masters RC, Lidzey D, Abrams KJ, Dapor M, Plenderleith RA, Rimmer S, Claeyssens F, Rodenburg C (2016): Angle selective backscattered electron contrast in the lowvoltage scanning electron microscope: Simulation and experiment for polymers. Ultramicroscopy 171, 126-138. https://doi.org/10.1016/j.ultramic.2016.09.006 